\title{
Una experiencia de flipped classroom aplicada a la docencia del Dibujo de Arquitectura
}

Caridad Yáñez, Eduardo; Fernández-Gago Longueira, Paula; Mantiñán Campos, Carlos

Escuela Técnica Superior de Arquitectura, Universidade da Coruña.

\section{RESUMEN}

La asignatura de Dibujo de Arquitectura agrupa la docencia del dibujo arquitectónico y del dibujo a mano alzada con el fin de introducir lo antes posible al alumnado en ambas vertientes del dibujo. La estructuración de la docencia de grado establecida por la UDC se ha demostrado ineficaz, después de varios años de aplicación, dadas las particularidades de cada una de las docencias, que además son impartidas con distintas metodologías. En el curso 2016-2017 se decide que para conseguir una mayor homogeneidad en los resultados entre los distintos subgrupos lo ideal sería una estructura en módulos de dos horas para cada metodología, incluyendo la teoría necesaria para cada una en su módulo correspondiente. Esa nueva estructura obligó a buscar alternativas para integrar adecuadamente las docencias expositiva e interactiva dentro de los nuevos márgenes temporales de dos horas. Durante el curso 2017-2018, para dar solución a la integración pretendida, el profesorado encargado de la docencia de dibujo arquitectónico constituyó un GIE con el objetivo citado, decidiendo impartir los contenidos teóricos correspondientes según el método de "flipped classroom" o clase invertida. En esta comunicación explicamos la experiencia.

PALABRAS CLAVE: flipped-classroom, clase-invertida, dibujo, arquitectura 


\section{CITA RECOMENDADA:}

Caridad Yáñez, E.; Fernández-Gago Longueira, P.; Mantiñán Campos, C. (2019): Una experiencia de flipped classroom aplicada a la docencia del Dibujo de Arquitectura. En De la Torre Fernández, E. (ed.) (2019). Contextos universitarios transformadores: construíndo espazos de aprendizaxe. III Xornadas de Innovación Docente. Cufie. Universidade da Coruña. A Coruña (pág. 149-160).

DOI capítulo: https://doi.org/10.17979/spudc.9788497497121.149

DOl libro: https://doi.org/10.17979/spudc.9788497497121

\section{ABSTRACT}

The subject of Drawing in Architecture, groups together the teaching of the architectural technical drawing and freehand drawing practice, with the purpose of introducing the students as soon as possible in both aspects of the architectural drawing. The organization of teaching in the Degree in Architecture established by the UDC, turned out to be ineffective after several years of application, given the special features of each part of the subject, which are also taught under different methodologies. In the 2016-2017 academic year, we decided that in order to achieve greater homogeneity in the results within the different subgroups, it would be interesting a two-hour module structure for each methodology, including the time required to teach the theoretical contents for each one. This new structure compelled us to look for alternatives to properly integrate the theoretical and practical teaching within the available two-hour schedule. During the past course 2017-2018, teachers responsible for the Supervised Projects methodology, constituted an Innovative Educational Work Group, with the previous aim, deciding to teach the corresponding theoretical contents according to the instructional strategy of flipped classroom. In this paper we explain the experience.

KEY WORDS: flipped-classroom, drawing, architecture 


\section{1. ¿PORQUÉ?}

La asignatura de Dibujo de Arquitectura (DA) del Grado en Estudios de Arquitectura, y anteriormente del Grado en Arquitectura, es el resultado de la reorganización en dos cuatrimestres de la antigua asignatura de Dibujo I, que agrupaba anualmente la docencia del dibujo arquitectónico y del dibujo a mano alzada.

Para conseguir introducir lo antes posible al alumnado en ambas vertientes del dibujo, la asignatura de DA comparte ambas docencias durante el primer cuatrimestre.

La estructuración de la docencia de grado establecida de manera genérica por la Universidade da Coruña (UDC) nos obligó a organizar la docencia en módulos de una hora de docencia expositiva y, en nuestro caso, tres horas de docencia interactiva donde la enseñanza del dibujo a mano alzada se desarrolla con una metodología de Taller y la del dibujo arquitectónico se lleva a cabo a través de Trabajos Tutelados.

Esta organización se mantuvo hasta el curso 2016-2017, cuando acordamos tomar dos decisiones de cara al curso siguiente, para conseguir una mayor homogeneidad en los contenidos, los resultados y las evaluaciones entre los distintos subgrupos. Por una parte, que los profesores de cada metodología fuesen distintos en cada subgrupo de prácticas, y por otra, establecer una estructura en módulos de dos horas para cada metodología, incluyendo la teoría necesaria para cada una de ellas en su módulo correspondiente.

Por ello la nueva estructura, aparte de significar una asignación doble de profesorado a cada subgrupo, un docente para Taller y otro para Trabajos Tutelados, implicó la búsqueda de alternativas para integrar adecuadamente las docencias expositiva e interactiva dentro de los nuevos márgenes temporales de dos horas.

Por otra parte, resulta obvio decir que la docencia del dibujo no es teórica. Los docentes podemos preparar clases magistrales "magníficas", pero la realidad, tozuda e inapelable, durante los años que hemos mantenido la estructuración establecida por la universidad nos ha demostrado lo siguiente: 
1. Que para el aprendizaje del dibujo, en cualquiera de sus vertientes, lo fundamental es su práctica por parte del alumnado. Sin ella no hay aprendizaje sustancial y significativo, por muy satisfechos que nos sintamos con las clases teóricas impartidas.

2. Que pretender mantener en las docencias del dibujo una dicotomía entre teoría y práctica no es lo adecuado. Claro que hay teoría, pero se trata de una teoría que debe acompañar a la práctica, de manera que vaya íntimamente ligada al fin que sirve: que no es otro que la representación adecuada de las formas y espacios arquitectónicos.

3. Que separar la teoría de la práctica empeora el resultado del aprendizaje, pues el alumnado al apreciar que va a ser evaluado por unos resultados gráficos (prácticos) de manera automática y equivocada, relega la teoría de sus prioridades dedicándose a una práctica que en consecuencia resulta menos eficaz.

4. Que el tiempo dedicado a las clases teóricas no es aprovechado por la mayoría de los estudiantes, que perciben las clases como el tiempo que les falta para concluir un partido de futbol claramente decidido, los minutos de la basura.

5. Que lo anterior resulta frustrante para los docentes que se han esforzado en preparar las mejores clases teóricas posibles que, finalmente, no son aprovechadas por una amplia mayoría de estudiantes.

Como resumen de lo anterior podemos relatar a modo de anécdota lo sucedido con un alumno, que en el desarrollo de una práctica realizó una pregunta acerca de una cuestión sobre la que la que incidimos mucho en las clases teóricas. La conversación fue similar a lo que podemos ver en la siguiente imagen: 


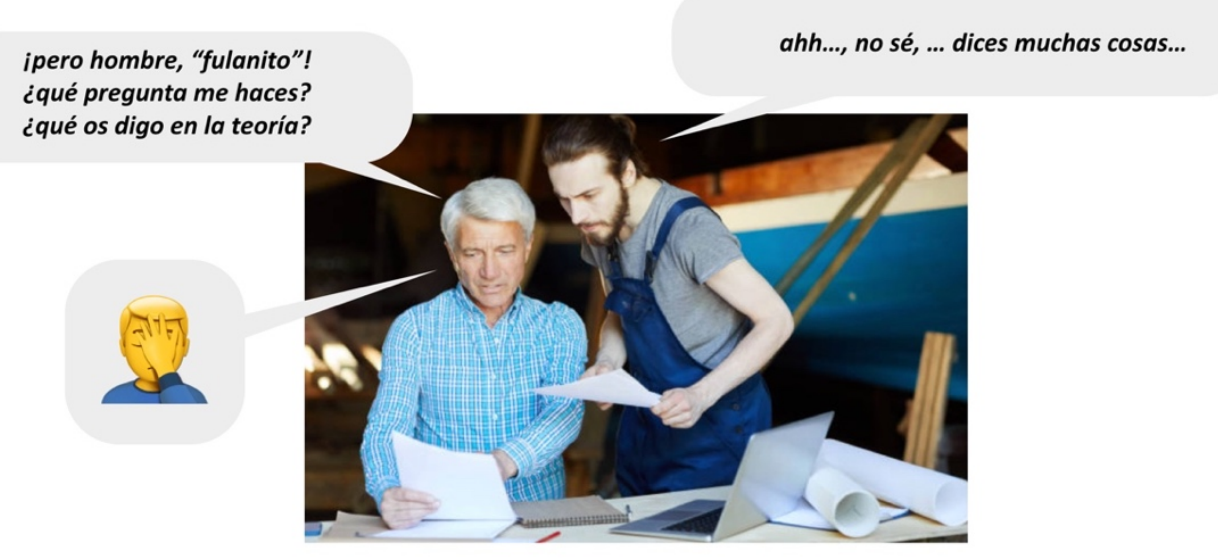

Imagen 1. Anécdota en clase

Esta anécdota, reafirmó nuestra percepción sobre como los estudiantes no asocian adecuadamente los contenidos teóricos con su aplicación en la práctica, así como la necesidad de buscar una alternativa a la dicotomía teoría-práctica impuesta por parte de la UDC en la implantación de los nuevos grados adaptados a Bolonia.

La alternativa buscada debe plantearse, necesariamente, como una integración de los contenidos teóricos en la práctica del dibujo, una teoría que obligatoriamente les llegue a los estudiantes, de manera previa y precisa, para que la puedan aplicar de una manera natural en la práctica.

\section{2. ¿CÓMO?}

Durante el curso 2017-2018, para dar solución a la integración pretendida, el profesorado encargado de la docencia de dibujo arquitectónico, dentro de la metodología de Trabajos Tutelados, constituyó un GIE con dicho objetivo, decidiendo impartir los contenidos teóricos correspondientes según el método de "flipped classroom" o clase invertida. 
La experiencia que hemos puesto en práctica parte de la lectura del artículo "Aprender al revés es más efectivo" de Ana Torres Menárguez, publicado en el País Digital.' En el que podemos leer:

“... La sensación de novedad que produce cualquier estímulo tiende a desaparecer después de 10 minutos. Con esa idea en la cabeza, Jon Bergmann (Chicago, 1964), reconocido en 2002 por la Casa Blanca como el mejor profesor de Estados Unidos de matemáticas y ciencias, rompió con la metodología de enseñanza tradicional. Dejó de basar sus clases en un discurso para no aburrir a sus alumnos y puso en marcha el llamado Flipped Classroom (en español, clase invertida). A partir de 2007, los estudiantes de química del instituto Woodland Park de Colorado empezaron a consumir la teoría en casa -con vídeos cortos hechos por el propio Bergmann- y a dedicar el tiempo de la clase a resolver dudas, investigar o trabajar por proyectos."

Según Bergman, el mayor atractivo del método es que posibilita los diferentes ritmos de aprendizaje del alumnado, sin dar por sentado que todos los estudiantes progresan de manera uniforme.

“...En un aula tradicional, el profesor cuenta una información que para algunos será muy fácil de procesar y para otros no. Luego van a casa a hacer los deberes y los que tienen unos padres con conocimientos de la materia, siempre salen ganando. Este método permite que en clase cada alumno reciba lo que necesita", explica Bergmann a EL PAÍS. Las conclusiones más contundentes de algunos de estos estudios inciden en que los estudiantes son más activos en clase, los profesores están más motivados y, en algunos casos, los alumnos obtienen mejores resultados en los exámenes.

Este método no es nuevo. En los setenta el pedagogo estadounidense Benjamin Bloom consideró erróneo dedicar la mayor parte del tiempo de las clases a escuchar una lección y dejar lo más creativo para casa. Según este teórico, es precisamente esa parte del aprendizaje la más complicada y la que requiere de un guía especializado, en este caso, el profesor... “ 
Todo ello se resume en la siguiente definición de flipped-learning ${ }^{\text {ii: }}$ El aprendizaje invertido es un enfoque pedagógico en el que la instrucción directa pasa del espacio de aprendizaje grupal al espacio de aprendizaje individual, y el espacio grupal resultante se transforma en un entorno de aprendizaje dinámico e interactivo donde el educador guía a los alumnos a medida que aplican conceptos y se involucran de manera creativa en el contexto de la materia.

Asumiendo este enfoque, nosotros pretendimos que los estudiantes adoptasen desde el primer día una actitud activa durante todo el proceso de aprendizaje, permitiendo que el tiempo presencial resultase más útil, para solucionar tanto dudas concretas derivadas de la lectura del material teórico avanzado como para rentabilizar el tiempo restante para el desarrollo parcial y la tutela de los trabajos prácticos planteados a lo largo del cuatrimestre.

En síntesis, el modelo se desarrolló de la siguiente manera: Semanalmente se seleccionaron los contenidos teóricos a impartir en ese período, y se sintetizaron de manera que la dedicación del alumnado a la lectura y asimilación de cada unidad teórica no supusiese más de 15-20 minutos de su tiempo no presencial previsto.

En este primer curso de implementación del método, los contenidos teóricos seleccionados se impartieron a través de ficheros "pdf" inéditos, con textos y gráficos especialmente diseñados para cumplir con la dedicación antes señalada. Este material se ha puesto a disposición de los alumnos a través de la plataforma Moodle. 


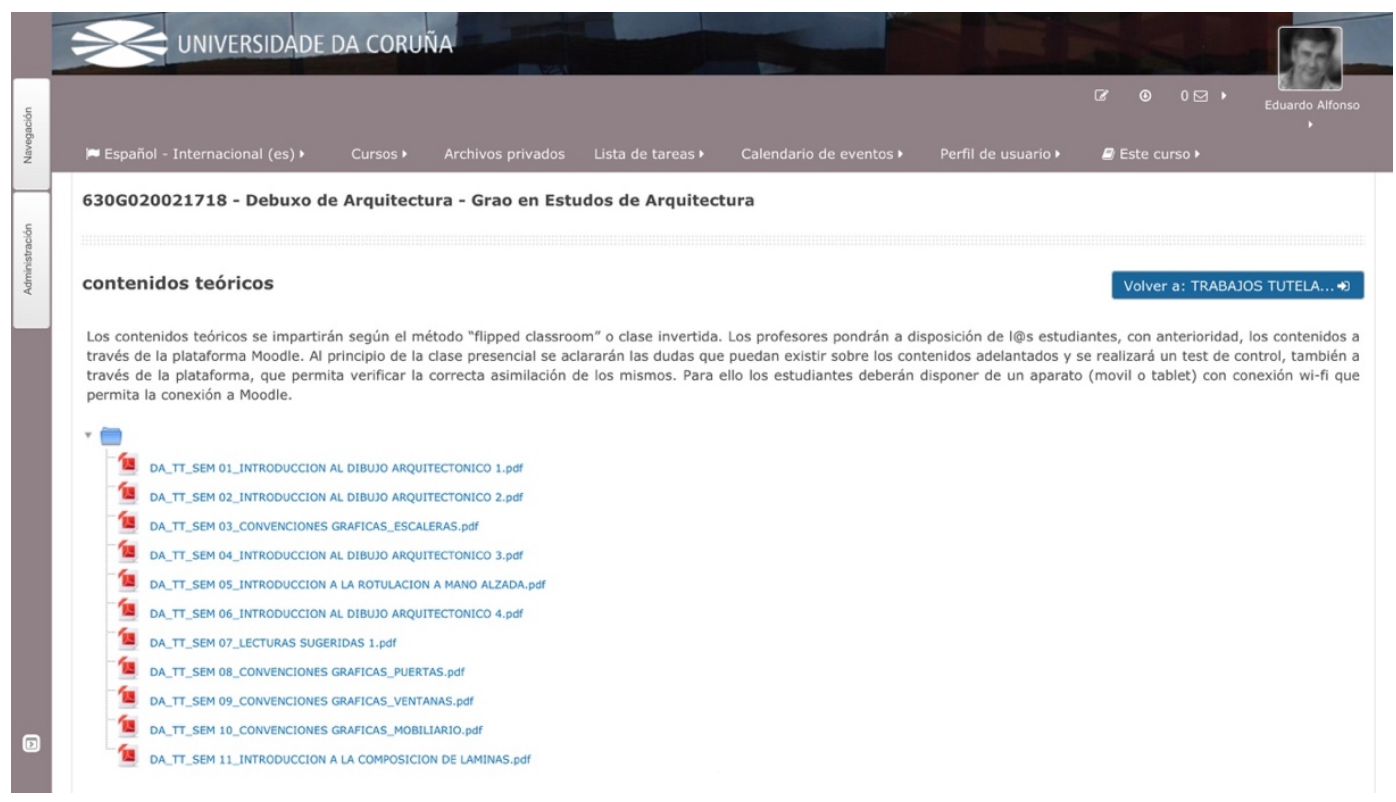

Imagen 2: Contenidos teóricos de la metodología de Trabajos Tutelados publicados en Moodle

Durante el tiempo no presencial, los estudiantes, debían leer y asimilar los contenidos teóricos publicados, además de ir realizando los Trabajos Tutelados propuestos.

A la semana siguiente, al principio de la clase, se comenzaba aclarando las dudas existentes sobre la unidad teórica avanzada para, a continuación, realizar un test de control a través de Moodle. Los test nos permitieron verificar la correcta asimilación de los contenidos por la mayor parte del alumnado.

En todo este proceso se emplearon una media de 15 minutos por sesión. De esta manera se han conseguido ganar 15 minutos de los 30 inicialmente disponibles para la teoría y que se dedican a la práctica. Además, durante el tiempo presencial destinado a práctica, los estudiantes continúan trabajando en los Trabajos Tutelados propuestos mientras el profesorado los supervisa de manera general, insistiendo en los aspectos principales de los contenidos teóricos. 


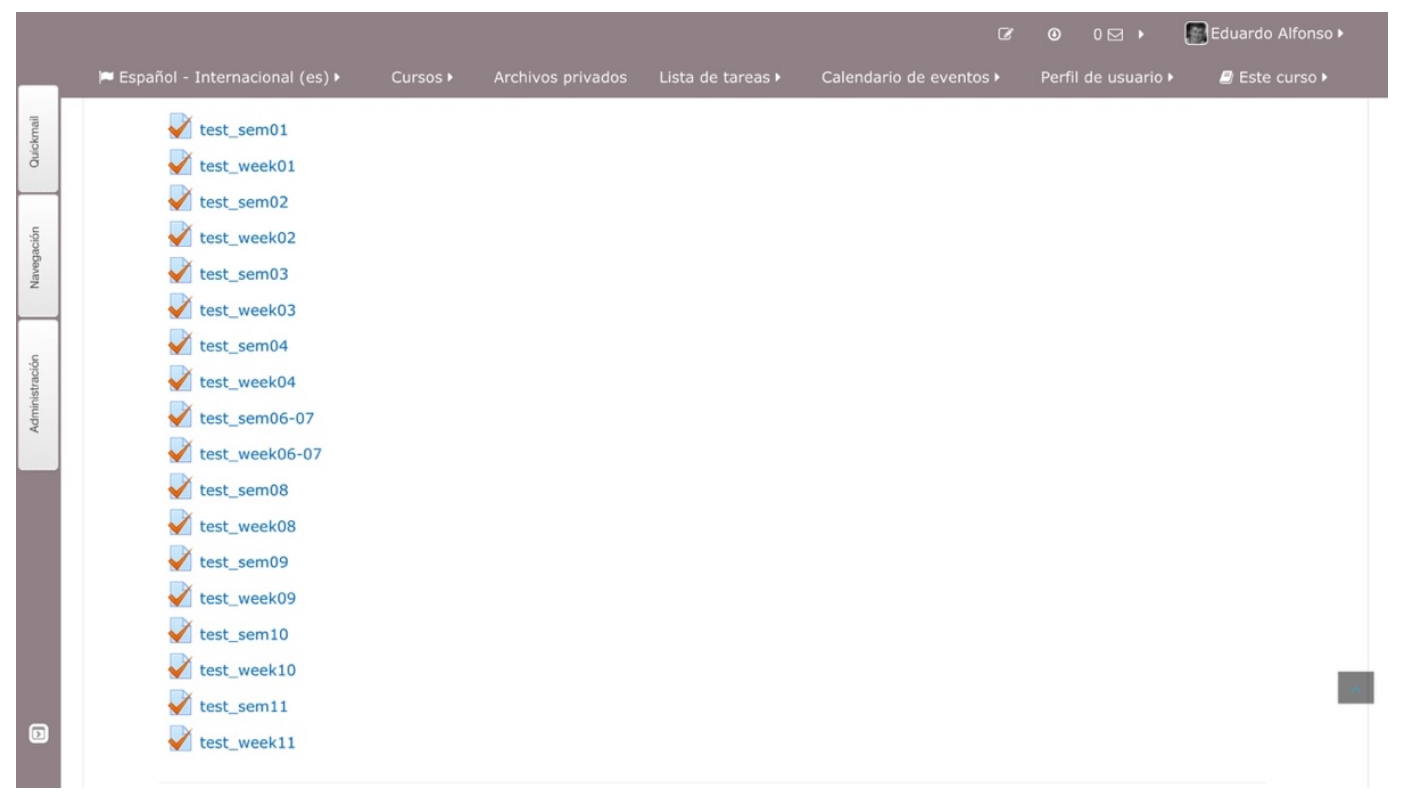

Imagen 3: Test realizados a través de Moodle sobre los conceptos avanzados en las distintas unidades teóricas

En el presente curso 2018-2019, se está llevando a cabo el refinado de los contenidos teóricos, perfeccionando aquellos que hemos visto que así lo requerían, ampliando algunos contenidos o reelaborando aquellos otros que consideramos necesario.

Para el curso 2019-2020 se pretende crear unos vídeos que recapitulen en 5 - 8 minutos los aspectos fundamentales de cada unidad semanal. Los textos ya revisados y aquilatados quedarán en Moodle, a modo de repositorio, para una mayor asimilación en caso de ser necesitado por algún estudiante.

\section{RESULTADOS}

Del primer año de aplicación del modelo solo podemos extraer resultados objetivos de los obtenidos a partir de los test. Aunque se facilitaron contenidos teóricos durante once semanas, solamente se realizaron nueve test, para no descabalgar grupos, debido a días festivos y tratando de que todos los grupos avanzasen a la par. 
De cada test, la plataforma Moodle nos ofrece los resultados obtenidos, lo cual nos permite hacer un estudio del mismo y sacar conclusiones. Así por ejemplo el test con menor número de suspensos (1) se corresponde con los contenidos de la semana 9, mientras que el test con mayor número de suspensos (13) se corresponde con los contenidos de la semana 4.

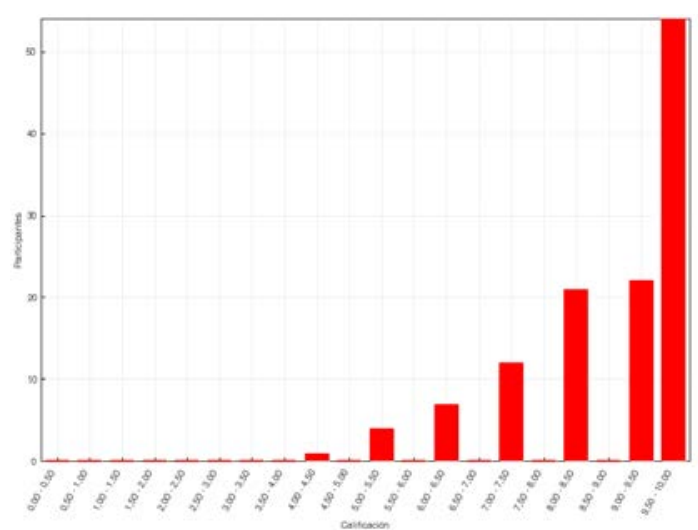

Resultados Test semana 9

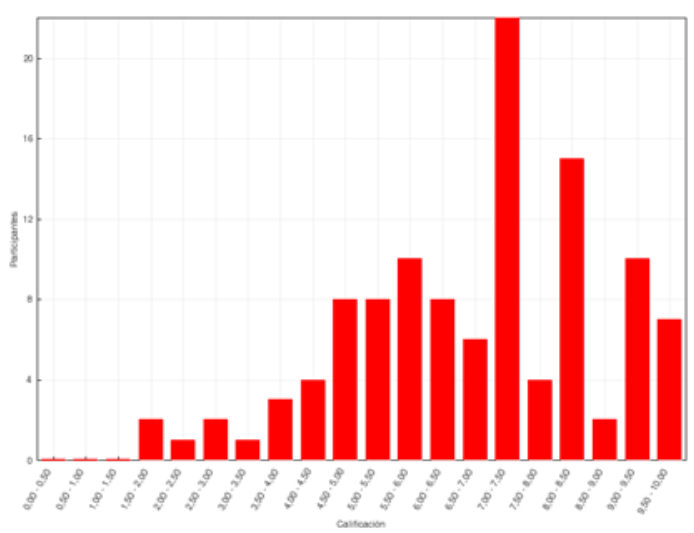

Resultados Test semana 4

Figura 1: comparación de los test con mayor y menor número de suspensos

Se presenta una imagen comparativa de los resultados en todos los test realizados, comparando los alumnos que aprobaron cada test, aquellos encuadrados por la trama azul, frente a los suspensos que se encuentran fuera de dicha trama. A simple vista, "peso visual", se puede apreciar que los resultados han sido buenos. 
resultados de los test semanales realizados
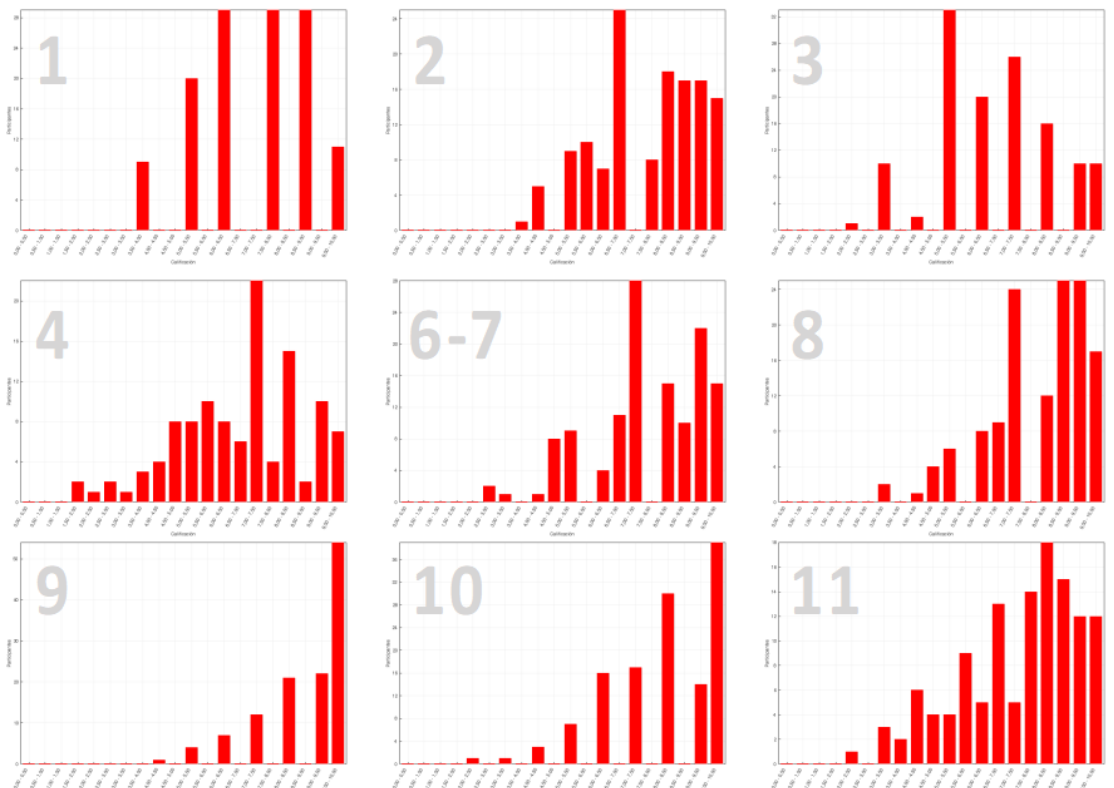

Figura 2: Comparativa de todos los test realizados, analizando el peso visual de los estudiantes aprobados dentro del área en azul.

Independientemente de los resultados pormenorizados obtenidos durante el primer año de aplicación del modelo, lo realmente interesante será poder establecer comparaciones con cursos venideros, así como las primeras conclusiones que se exponen a continuación.

\section{CONCLUSIONES}

Con este modelo hemos conseguido que la mayoría de los estudiantes abandonen su dejadez hacia los contenidos teóricos y adopten una actitud proactiva hacia la teoría desde el primer día, logrando que la incorporen como algo esencial al proceso de aprendizaje práctico.

Hemos garantizado que el alumnado asimile los contenidos teóricos adecuadamente, aspecto imposible de alcanzar anteriormente al ser la evaluación fundamentalmente práctica. 
Asimismo, hemos logrado que el tiempo presencial sea más útil, tanto para solucionar dudas concretas derivadas de la asimilación del material teórico avanzado en cada unidad semanal, como para rentabilizar el tiempo restante de clase práctica presencial, empleándolo en el desarrollo de los Trabajos Tutelados propuestos, compensando de esta manera el tiempo que los estudiantes destinan a la lectura y comprensión en casa de los contenidos teóricos publicados en Moodle.

Por último, como conclusión final, consideramos que este modelo resulta muy adecuado para impartir la teoría en asignaturas de desarrollo fundamentalmente práctico.

\section{REFERENCIAS BIBLIOGRÁFICAS}

Torres Menárguez, Ana. "Aprender al revés es más efectivo". El País Digital, (2016). https://elpais.com/economia/2016/10/28/actualidad/1477665688_677056.html

Blog de Red de Aprendizaje Invertida: https://flippedlearning.org/definition-of-flipped-learning/

' Torres Menárguez, Ana (2016), Aprender al revés es más efectivo. El País Digital. Consultado en: https://elpais.com/economia/2016/10/28/actualidad/1477665688_677056.html

ii Flipped Learning Network (FLN). (2014) The Four Pillars of F-L-I-P ${ }^{\text {тм }}$. Reproducible PDF consultado en: https://flippedlearning.org/definition-of-flipped-learning/ 